\title{
STUDY OF NOISE-INDUCED TRANSITIONS IN THE LORENZ SYSTEM USING THE MINIMUM ACTION METHOD*
}

\author{
XIANG ZHOU $^{\dagger}$ AND WEINAN E
}

Dedicated to the sixtieth birthday of Professor Andrew Majda

\begin{abstract}
We investigate noise-induced transitions in non-gradient systems when complex invariant sets emerge. Our example is the Lorenz system in three representative Rayleigh number regimes. It is found that before the homoclinic explosion bifurcation, the only transition state is the saddle point, and the transition is similar to that in gradient systems. However, when the chaotic invariant set emerges, an unstable limit cycle continues from the homoclinic trajectory. This orbit, which is embedded in a local tube-like manifold around the initial stable stationary point as a relative attractor, plays the role of the most probable exit set in the transition process. This example demonstrates how limit cycles, the next simplest invariant set beyond fixed points, can be involved in the transition process in smooth dynamical systems.
\end{abstract}

Key words. Noise-induced transitions, Lorenz system, limit cycle, transition set, minimum action path.

AMS subject classifications. $\quad 34 \mathrm{D} 10,82 \mathrm{C} 35,82 \mathrm{C} 26$.

\section{Introduction}

In recent years, there has been a great deal of progress in the study of weak noise-induced transition in equilibrium systems whose dynamics are determined by gradient flows $[1,2,3,4]$. One main feature in these systems is that in the limit as the noise amplitude goes to 0 , the so called most probable escape path (MPEP) must approach a saddle point on the separatrix of the unperturbed system. Such a saddle point with only one unstable direction is thus the most probable exit point or the transition state. At the same time, it has been realized that non-gradient systems or systems that lack of the property of time reversibility can give rise to a large variety of new phenomena. Examples of these systems range from Josephson junctions [5] and switching in lasers [6] to protein folding [7] and electronic circuits [8]. Some of the most interesting findings include a preexponential factor of the Kramers rate and the transition state of an unstable point [9], a symmetry breaking bifurcation of the optimal escape path [10], and the phenomenon of cycling of exit point distribution from a planar unstable limit cycle [11].

One of the fundamental questions in the study of the noise-induced transitions between metastable states is what kind of new transition states appear in the perturbed non-gradient systems, and how to find them. The identification of the optimal transition path is very useful. The optimal transition path is where the distribution of the fluctuational trajectories which make successful transitions between attractors sharply peaks around. The optimal transition path is deterministic in spite of the stochastic nature of the transition process. In gradient systems, the optimal transition path is always parallel to the drift $b=-\nabla V$ and the MPEP is the time reversed

\footnotetext{
*Received: November 14, 2008; accepted (in revised version): March 7, 2009. This work was partially supported by ONR grant N00014-01-1-0674, DOE grant DOE DE-FG0203ER25587, NSF grants DMS-0708026 and DMR-0611562.

${ }^{\dagger}$ Program for Applied and Computational Mathematics, Princeton University, Princeton, NJ 08544, USA (xiangz@math.princeton.edu).

$\ddagger$ Department of Mathematics and Program for Applied and Computational Mathematics, Princeton University, Princeton, NJ 08544, USA (weinan@math.princeton.edu).
} 
heteroclinic trajectory of the unperturbed system connecting the attractor and the saddle point $[12,2]$. The generic feature of the optimal transition path between two attractors is that it approaches certain transition state (such as saddle points in gradient systems) on the basin boundary of one attractor, then it runs along the unstable manifold of that transition state and enters the basin of attraction of the second attractor. The optimal transition path provides lots of information for the transition mechanism. Once it is known, one can analyze the path to identify transition states in the phase space.

There has been some theoretical progress on finding the optimal transition path and the transition states in some examples of non-gradient systems. Even though many non-gradient systems still possess saddle points (with one positive eigenvalue) as transition states, they are not the only possible transition states, even in a simple planar system without a limit cycle [13], where the MPEP asymptotically approaches an unstable fixed point on the separatrix. In the case of a limit cycle, which itself is the basin boundary in a planar system, the MPEP spirals toward the limit cycle asymptotically and its $\omega$-limit set is the complete limit cycle [11].

Meanwhile, there has been much work on the two-dimensional discrete maps perturbed by weak white noise by using statistical analysis of the sampled transition trajectories. These works $[14,15,16,17,18,19,20,21,22]$ cover a variety of classical discrete map models. In these examples, the invariant sets involved in transition processes include primary homoclinic tangencies within nonhyperbolic chaotic attractors [16] and saddle periodic orbits within some chaotic attractors or on fractal basin boundaries [22]. Based on a small number of numerical examples, it was conjectured [17] that in the noise-induced escape from the basin of attraction of an invariant set, the chaotic behavior of low-dimensional dynamical systems is largely determined by the position and stability properties of unstable cycles built into the chaotic structures.

All of the above findings suggest that in general nongradient systems, it is not a single saddle point that acts as a transition state, but generally involves other types of invariant sets, such as periodic orbits, or an unstable manifold of an unstable stationary point on the separatrix [13]. These invariant sets either lie on the regular separatrix or are embedded in fractal basin boundary. When transitions take place, the transition trajectories cross these invariant sets with dominated probability compared with crossing other parts on the separatrix. We call such invariant sets as most probable exit invariant sets or transition sets.

Up to now, the investigations of higher dimensional smooth (autonomous) dynamical systems (such as PDE systems in [23, 24]) do not show any new examples of transition sets except saddle points. We investigate the Lorenz system in this paper, where the chaotic invariant set emerges. On the other hand, from a numerical perspective, identifying the transition states or transition sets in an arbitrary dynamical system remains a serious challenge. We will make use of the minimum action method [23], which is based on the Freidlin-Wentzell theory [25], to identify the optimal transition path. We are going to report the transition paradigms between two symmetric stable fixed points in the weak noise perturbed Lorenz system with three representative values of the Rayleigh number $\rho$. These three cases account for the three representative phase space structures in the Lorenz system when the two symmetric fixed points still maintain their stabilities. We find that the transition process is similar to that in gradient systems before a homoclinic explosion bifurcation $\rho<\rho_{t} \approx 13.92$. However, when the chaotic invariant set emerges in the Lorenz system, a pair of symmetric unstable limit cycles $\left(L_{ \pm}\right.$in figure 2.1$)$ continues from 

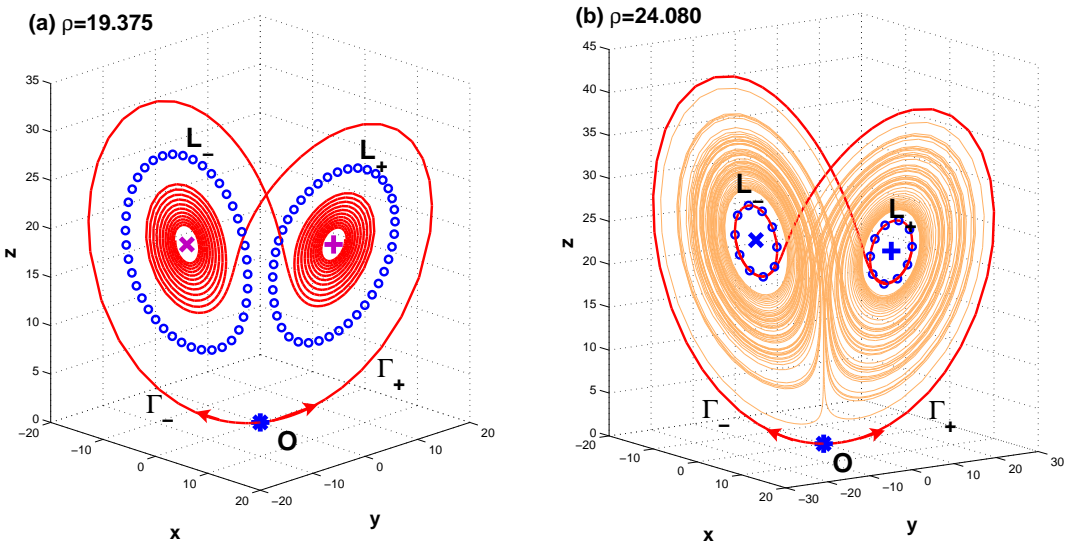

FIG. 2.1. The Lorenz system with two different values of the Rayleigh number $\rho$. $\Gamma_{ \pm}$(red curve) is the unstable manifold of the origin $O$. The pair of the simplest limit cycles $\left(L_{+}\right)$belonging to the strange invariant set $\Lambda$ are labelled by the isolated blue circles. " $\times$ " and "+" show two nontrivial stationary points, $q_{-}$and $q_{+}$respectively. In panel (b), a trajectory in the Lorenz attractor is colored in brown to illustrate the position of the Lorenz attractor.

the homoclinic trajectory. One of these two limit cycles, which is embedded in a local tube-like manifold around the initial stable stationary point as a relative attractor, plays a key role in the transition process. The optimal transition path escapes from the initial stable stationary point by approaching this limit cycle and then enters the chaotic invariant set. The transition path continues to approach a saddle point, the origin, which is a second transition state. Then the path moves towards the other stable stationary point. There is a slight difference between the situations of transient chaos $\rho<\rho_{a} \approx 24.06$ and chaotic attractor $\rho>\rho_{a}$, but the transition set, the limit cycle, and the transition state, the saddle origin, are both present in these two transition processes. It suggests that limit cycles, the next simplest invariant set beyond the fixed points, can in general be involved in the transition process in smooth dynamical systems.

The paper consists of five sections. The basic results on the Lorenz system and its phase space structure are reviewed in section 2 . In section 3 , we review the minimum action method since it is our basic tool. The main results about the transition paradigms in the Lorenz system are presented and discussed in detail in section 4 . Conclusions are drawn in the last section.

\section{The Lorenz system and its phase space structures}

\begin{tabular}{|l|l|l|}
\hline$\rho=1$ & pitchfork bifurcation & $\begin{array}{l}\text { the origin } O \text { loses 1-dim stability; } \\
\text { two stable stationary points } q_{ \pm} \text {arise. }\end{array}$ \\
\hline$\rho_{t} \approx 13.92$ & $\begin{array}{l}\text { homoclinic explosion } \\
\text { preturbulence }[26]\end{array}$ & $\begin{array}{l}W^{u}(O) \subset W^{s}(O) \text { : homoclinic orbits; } \\
\text { transient chaos arise }\left(L_{ \pm} \text {appear). }\right.\end{array}$ \\
\hline$\rho_{a} \approx 24.06$ & $\begin{array}{l}\text { bifurcation to Lorenz attractor } \\
\text { heteroclinic orbits }\end{array}$ & $\begin{array}{l}\left.W^{u}(O) \subset W^{s}\left(L_{ \pm}\right) \text {(refer to figure } 2.1\right) ; \\
\text { chaotic attractor arise ("butterfly" shape). }\end{array}$ \\
\hline$\rho_{h} \approx 24.74\left(\frac{470}{19}\right)$ & subcritical Hopf bifurcation & $L_{ \pm}$disappear and $q_{ \pm}$lose stability. \\
\hline
\end{tabular}

TABLE 2.1. Bifurcations in the Lorenz system 
The example we investigate in this paper is the Lorenz system:

$$
\left\{\begin{array}{l}
\dot{x}=\sigma(y-x), \\
\dot{y}=\rho x-y-x z, \\
\dot{z}=-\beta z+x y .
\end{array}\right.
$$

This model has a natural symmetry $(x, y, z) \rightarrow(-x,-y, z)$ for all values of the parameters. As usual, we fix the parameters $\sigma=10, \beta=\frac{8}{3}$. Table 2.1 briefly summarizes the bifurcations in this system and figure 2.1 shows two pictorial descriptions of the phase space. More detailed analysis and discussions can be found in the books $[27,28]$ and other references $[29,30,31,26]$. The global stable manifolds in the Lorenz system were analyzed and visualized in $[32,33,34,35]$.

According to Table 2.1, we choose three representative values of the Rayleigh parameter $\rho: \rho_{1}=10, \rho_{2}=19.375$ and $\rho_{3}=24.08$. In this regime, the two nontrivial stationary points

$$
q_{ \pm}=( \pm \sqrt{\beta(\rho-1)}, \pm \sqrt{\beta(\rho-1)}, \rho-1)
$$

are stable and the origin $O$ is a saddle point with one dimensional unstable manifold $W^{u}(O)$ and two dimensional stable manifold $W^{s}(O)$. The one dimensional manifold $W^{u}(O)$ has two branches $\Gamma_{+}$and $\Gamma_{-}\left(\Gamma_{+}\right.$is defined to point to the direction of positive values of $x$ and $y$ and $\Gamma_{-}$is the symmetric image of $\left.\Gamma_{+}\right)$.

The phase space structures for $\rho_{1}, \rho_{2}$ and $\rho_{3}$ can be summarized as follows.

1. $\rho_{1}=10$. This is the simplest case. The whole phase space is divided by $W^{s}(O)$ into two basins of attractions of the stable stationary points $q_{-}$and $q_{+}$, i.e., the separatrix of $q_{ \pm}$is the two-dimensional surface $W^{s}(O)$ and the saddle point $O$ is the unique fixed point on this manifold. All trajectories on the separatrix $W^{s}(O)$ go to this saddle point $O$ (thus, this saddle point is called relative attractor in some references). The unstable manifold $W^{u}(O)$ has two branches $\Gamma_{+}$and $\Gamma_{-}$which are heteroclinic orbits, from the origin $O$ to the stationary point $q_{+}$and $q_{-}$, respectively. There is no limit cycle or chaotic invariant set at this value $\rho_{1}$.

2. $\rho_{2}=19.375$. If $\rho=\rho_{t}$, then each of $\Gamma_{ \pm}$forms a homoclinic orbit at the origin. When $\rho$ passes this value, e.g., at $\rho=\rho_{2}, \Gamma_{ \pm}$wind around the $z$-axis and then spiral to $q_{\mp}$ respectively, instead of $q_{ \pm}$. The phase space undergoes a dramatic change. A hyperbolic invariant set $\Lambda$ of saddle type is identified by the Lorenz geometric model. The symbolic description of $\Lambda$ is similar to that of Smale's horseshoe. $\Lambda$ contains a countable number of periodic orbits of arbitrarily long periods as well as an uncountable number of bounded aperiodic orbits, generated from the homoclinic explosion. Two simplest periodic orbits $L_{ \pm}$ belonging to the invariant set $\Lambda$, with symbols $\overline{0}$ and $\overline{1}$ (cf. [27]), are shown in figure 2.1. $L_{ \pm}$wind around the nontrivial stationary points $q_{ \pm}$, respectively. These two periodic orbits are of saddle type. Both their geometric sizes and periods shrink as $\rho$ increases further and they collapse onto $q_{ \pm}$at the subcritical Hopf bifurcation point $\rho_{h}$ where $q_{ \pm}$become saddle points. The Lebesgue measure of the invariant set $\Lambda$ is zero and almost all trajectories ultimately tend to one of the two sinks $q_{+}$or $q_{-}$. Yet the transient time to reach $q_{+}$or $q_{-}$is very long and highly sensitive to the starting points of the trajectories. Hence, this is called transient chaos, or preturbulence [26]. The hyperbolic set $\Lambda$ separates regions of phase space which have different patterns 
of revolutions about $q_{+}$and $q_{-}$before they reach a small neighborhood of one of these points and "settle down". These two regions stagger each other to make the basin boundary fractal (cf. figure 4.5).

3. $\rho_{3}=24.08$. At this value, a strange attractor arises while the stationary points $q_{ \pm}$are still stable since the Hopf bifurcation does not occur yet. The strange attractor, the Lorenz attractor, is emerged from the invariant set $\Lambda$ discussed above. We still denote this strange attractor by $\Lambda$. However, this Lorenz attractor is different from the original strange invariant set at $\rho_{2}$ because the orbits in the Lorenz attractor will never set down to any fixed point or periodic orbit. The Lorenz attractor $\Lambda$ consists of a Cantor set of twodimensional sheets, excluding a neighborhood of the stable stationary points $q_{ \pm}$. The saddle cycles $L_{ \pm}$are not part of the attractor and their stable manifolds separate the trajectories that approach $q_{ \pm}$from those that approach the Lorenz attractor $\Lambda$.

\section{The Minimum action method}

Methods for finding optimal transition paths can be divided into two categories: Monte Carlo methods and variational methods. Monte Carlo simulation solves the underlying stochastic differential equation for sufficiently long time so that a large number of realizations of the transition events can be collected for statistical analysis. The approach for constructing prehistory distribution in [36] relies on this method. It has been extensively applied to low-dimensional problems, such as those in [37]. Variational methods [23] are based on the Freidlin-Wentzell theory which states that the optimal transition path in zero noise limit can be characterized as the minimizer of the Freidlin-Wentzell action functional which has the following form [25],

$$
S_{T_{1}, T_{2}}[\varphi]=\frac{1}{2} \int_{T_{1}}^{T_{2}}|\dot{\varphi}(t)-b(\varphi(t))|^{2} d t
$$

where $\varphi(t)$ is an absolutely continuous function in $C_{\left[T_{1}, T_{2}\right]}$ and $b$ is the vector field of the unperturbed dynamical system.

An auxiliary Hamiltonian system associated with the Freidlin-Wentzell functional can be derived:

$$
\left\{\begin{array}{l}
\dot{\varphi}=b(\varphi)+p \\
\dot{p}=-(\nabla b)^{T} p,
\end{array}\right.
$$

where the Hamiltonian $H(\varphi, p)=\langle b(\varphi), p\rangle+\langle p, p\rangle / 2$. The solutions of the auxiliary Hamiltonian ODE with the value of the Hamiltonian being zero are called zero-energy trajectories. According to the Freidlin-Wentzell theory, the MPEP (which may not be unique in some cases) is the zero-energy trajectory that escapes from the attractor and has the least action $S^{*}=\min _{H(\varphi, p)=0} S[\varphi]$ when $\varphi$ escapes the basin of attraction. The escape action can be viewed as a function of a set of parameters characterizing the initial conditions of the zero-energy trajectories. A topological method based on this fact, which is essentially a shooting method, was suggested in [38] and applied to two dimensional continuous time dynamical systems and discrete maps. The application of this approach in general dynamical systems is severely limited by the difficulties associated with parametrizing all zero-energy trajectories and finding an efficient search strategy to explore the parameter space, as well as the unknown boundary condition where the MPEP exits the basin boundary. 
An alternative numerical algorithm, the minimum action method ("MAM"), was proposed in [23] which directly minimizes the Freidlin-Wentzell functional. In MAM, the transition path is a curve which minimizes the Freidlin-Wentzell functional (3.1). The path obtained in this way is called minimum action path ("MAP"). If the dynamical system is a gradient system, the minimum action path has a simpler geometric characterization (the minimum energy path, or "MEP") and a simplified algorithm, the string method developed in [3,4], works better for this case. There have been some recent improvements of MAM: the adaptive minimum action method ("aMAM", [39]) and the geometric minimum action method ("gMAM", [40]). Both are designed to overcome the difficulties associated with representing the optimal path using the physical time. The gMAM is based on a reformulation of the Freidlin-Wentzell functional in the arclength parametrization. The aMAM uses the original physical time to parametrize the transition path but incorporates the moving mesh strategy. In the end, both methods allow us to optimally distribute the grid points at places where the transition takes place.

One potentially tricky point is the choice of the parameter $T_{1}$ and $T_{2}$. In principle, we want to choose them to be infinite. In practice, we need to use finite values. A nice thing is that the error due to finite truncation drops very fast (nearly exponentially) with increasing $T_{2}-T_{1}$ [39]. In our computations for the Lorenz system, we tested different transition times and numbers of mesh points to ensure that our numerical results are robust. The results presented here correspond to the transition time $T_{2}-$ $T_{1}=80$ and the number of mesh points is 2000 for the case with $\rho_{1}$ and 4000 for the cases with $\rho_{2}$ and $\rho_{3}$. These are sufficient for the features that we report below. In our computation for the MAP, we found that higher accuracy was necessary for the parameter values $\rho_{2}$ and $\rho_{3}$ than for the value $\rho_{1}$ to capture the shape of the path along $\Gamma_{-}$(see Section IV). One main source of error comes from insufficient resolution of the saddle point $O$. Once we are assured that the saddle point $O$ is a transition state, we split the MAP from $q_{-}$to $q_{+}$into two parts: from $q_{-}$to $O$ and from $O$ to $q_{+}$ and run the MAM separately for each part. This simple trick gives us more accurate paths for the $\rho_{2}$ and $\rho_{3}$ cases.

\section{Transition Paradigms in the Lorenz model}

We now report our findings for the transition processes between the two equilibria $q_{ \pm}$of the Lorenz system (2.1) with weak additive white noise,

$$
\left\{\begin{array}{l}
\dot{x}=\sigma(y-x)+\sqrt{\varepsilon} d W_{t}^{x}, \\
\dot{y}=\rho x-y-x z+\sqrt{\varepsilon} d W_{t}^{y}, \\
\dot{z}=-\beta z+x y+\sqrt{\varepsilon} d W_{t}^{z} .
\end{array}\right.
$$

4.1. $\rho=\rho_{1}$. $\quad$ Figure 4.1 shows the numerically calculated minimum action path (MAP). This path has a sharp turn at the saddle point $O$, which divides the path into two segments. The first one is the MPEP and the second one coincides with the heteroclinic orbit $\Gamma_{+}$. Our next quantitative analysis of the MAP is presented in figure 4.2. Fig. 4.2a and figure 4.2b confirm that only one single point, the saddle $O$, separates the MAP into two segments which lie in basins of attraction of $q_{-}$and $q_{+}$, respectively. figure $4.2 \mathrm{c}$ is the plot of the amplitude of the noise $\|\dot{\varphi}-b(\varphi)\|$, reflecting the contributions of the fluctuational noise for the system to escape from the basin of attraction of $q_{-}$. Thus, figure 4.2 provides solid evidences to the fact that the origin $O$, the unique saddle point in the phase space is exactly the location where the optimal transition path crosses the basin boundary, i.e., the saddle point $O$ is the transition state, which is very similar to that of gradient systems. The transition process here 


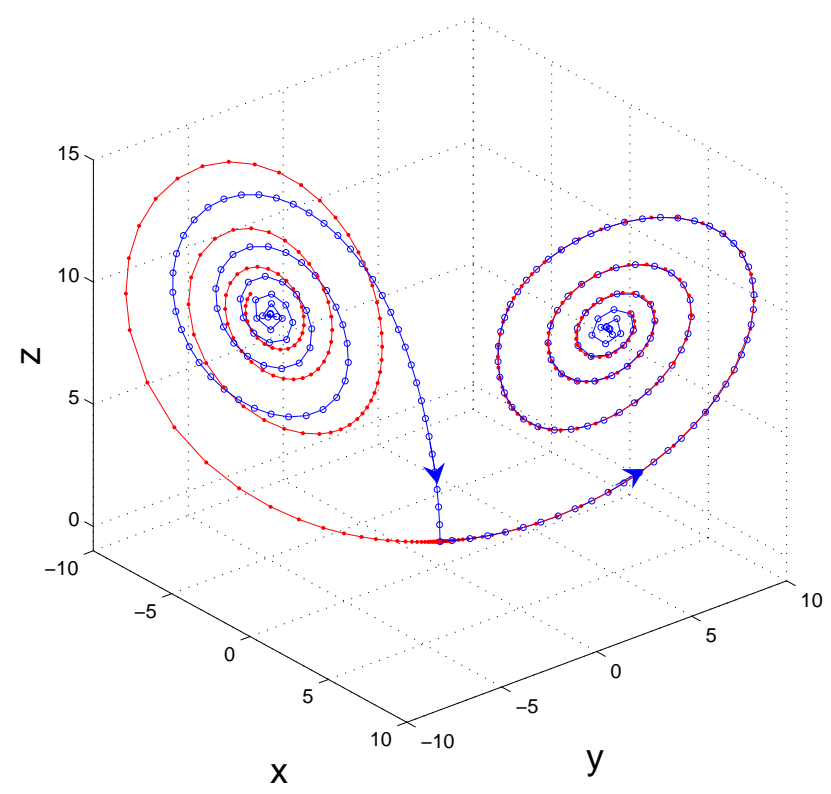

FIG. 4.1. The simple bistable case $(\rho=10)$ : the MAP (blue curve, open circles) spirals out from the initial state $q_{-}$and lands toward the final state $q_{+}$spirally, exactly passing the saddle point (the origin). The heteroclinic orbits $\Gamma_{ \pm}$(red curve, filled circles) starting from the origin $O$ are also shown for comparison.

can be viewed as simply as " $q_{-} \rightarrow O \rightarrow q_{+}$" in this bistable system, although the global structure of the separatrix of the Lorenz system, i.e., $W^{s}(O)$, is "pleat"-like and "twisted", especially near the $z$ axis or away from the origin (cf. figure 11 and figure 12 in [32]).

4.2. $\rho=\rho_{2}$ : transient chaos. The numerical MAP in this case is plotted in figure 4.3. The first observation by measuring the distance between the MAP and the point $O$ as before, is that the MAP still goes through the saddle point $O$. Refer to figure 4.4c. After passing the saddle point, the path follows the orbit $\Gamma_{-}$to approach $q_{+}$. This reflects the fact that at the homoclinic bifurcation when $\rho$ passes $\rho_{t}$, the heteroclinic trajectory from $O$ to $q_{+}$makes a change from $\Gamma_{+}$to $\Gamma_{-}$. A sample of fluctuational transition path from Monte Carlo simulation is plotted in figure 4.3 to suggest one possibility of the transition process. The numerical discrepancy at $\Gamma_{-}$near the origin $O$ between the sample path and the MAP is due to the very strong expansion rate of the Lorenz system along $\Gamma_{-}$and the finite noise intensity. It may suggest that the distribution of the fluctuational transition trajectories is nonGaussian and skewed on one side of the MAP, instead of centering along the MAP. This is a generic feature for non-gradient systems [41].

A new phenomenon arises in the life time plot of figure 4.4. There is a segment in the middle of the MAP showing extremely high sensitivity of life time. We mark this segment of the MAP by two ends $A$ and $B$ in figure 4.3 and call it "chaotic" life time segment for convenience. The part of the MAP before the point $A$ is completely in the basin of attraction of $q_{-}$. The appearance of this "chaotic" life time segment after the point $A$ implies that there is a qualitative change in the phase space near 
(a) life time

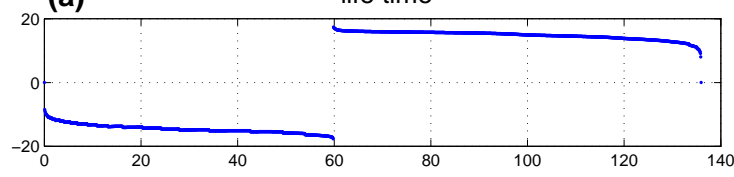

(b) distance to the origin

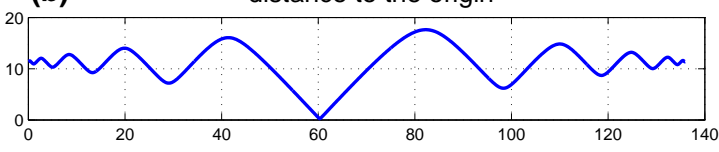

(c) the optimal fluctuational force

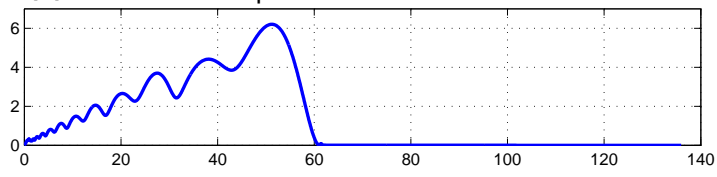

FIG. 4.2. (a) The life time plot for each point on the MAP in figure 4.1. The horizontal axis is the arclength parameter of the MAP. The sharp jump point in the top figure corresponds to the origin $O$. The life time is defined to be the (signed) time in the original dynamical system to reach the $\alpha$-neighborhood of the final state (positive life time) or the initial state (negative life time). $\alpha=5 \times 10^{-4}$ in our numerical experiments. This value guarantees that, for all $\rho_{1}, \rho_{2}$ and $\rho_{3}$ considered in this paper, the $\alpha$-neighborhood is always completely included in the basin of attraction of the stable stationary point inside of it. (b) the Euclid distance to the origin $O$ from points on the MAP. (c) The $L_{2}$ norm of the optimal fluctuation force $\left\|\varphi_{t}-b(\varphi)\right\|_{2}$ (b is the vector field of the Lorenz system) for each point on the MAP $\varphi$.

the point $A$ and the curve $A B$ travels through the chaotic invariant set $\Lambda$.

We want to break down how "chaotic" life time segment of the MAP enters the set $\Lambda$ at the point $A$. By using Newton's method in [42] to search for limit cycles, we find that this point $A$ is located on a limit cycle $L_{-}$(cf. figure 4.4b). This limit cycle $L_{-}$was introduced in Section II. It is of saddle type and has a two-dimensional stable manifold and a two-dimensional unstable manifold. Thus, it is also called saddle cycle. It is the most probable invariant set, or transition set, from the stationary point $q_{-}$ to the transient chaos $\Lambda$. The complete transition process from $q_{-}$to $q_{+}$therefore can be viewed as a successive series of steps: " $q_{-} \rightarrow L_{-} \rightarrow O \rightarrow q_{+}$".

A few remarks are in order. The first is about the point $A$, which is also the intersection point of the numerical MAP and the saddle cycle $L_{-}$(cf. figure 4.3c). It should be distinguished from the most probable exit point on a limit cycle when a small but finite amount of noise is present, which has been examined both analytically $[43,11]$ and numerically [44] for a planar system, the inverted van de Pol system with the noise amplitude $\varepsilon$. The authors there discovered an oscillatory behavior or cycling effect, namely, that for each given noise amplitude $\varepsilon$, there is a most probable exit location $A_{\varepsilon}$ on the limit cycle and this location $A_{\varepsilon}$ (the peak of the exit distribution on the limit cycle) slowly oscillates along the limit cycle with the period $\sim|\log \varepsilon|$ as $\varepsilon \downarrow 0$. In the zero-noise limit, the MPEP spirals outward to the complete limit cycle asymptotically and never intersects with the limit cycle. It is actually the heteroclinic orbit between the stable fixed point and the limit cycle in the augmented Hamiltonian system. The arclength of the MPEP is thus infinite. In our example, the intersection point $A$ here in figure 4.3 is a numerical artifact due to the finite length 


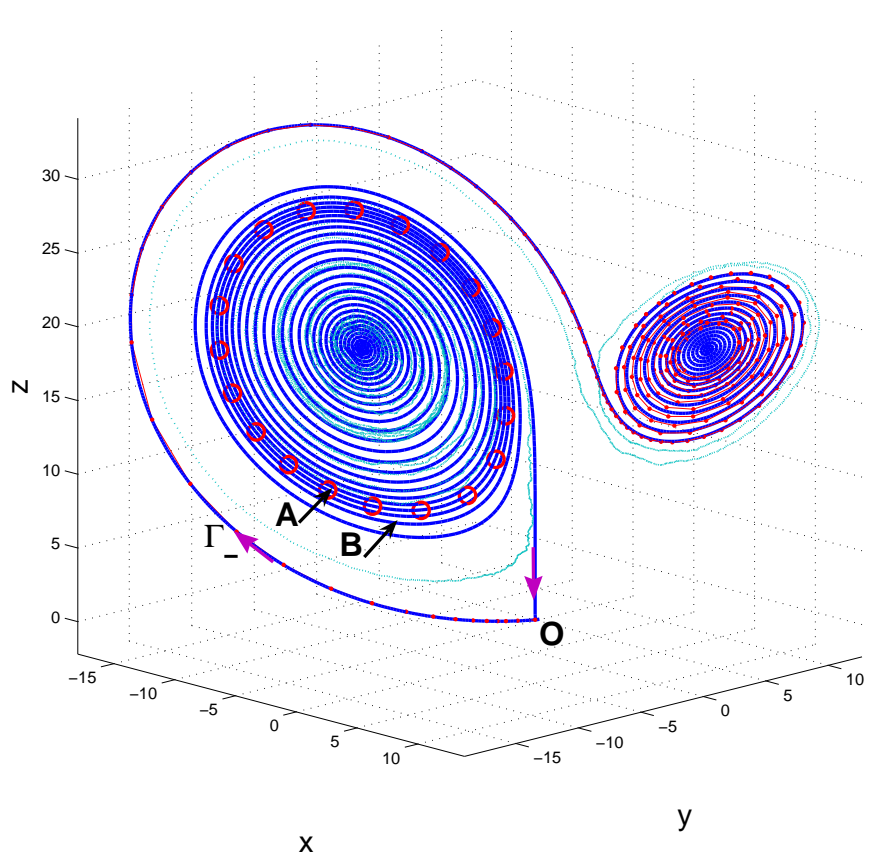

FIG. 4.3. The transient chaos case $(\rho=19.375)$ : the MAP is the blue solid curve. A realization of the transition path with $\varepsilon=0.01$ is also shown as a dotted grey curve. The red curve with small solid dots is $\Gamma_{-}$connecting the origin $O$ and the stationary point $q_{+}$. The big open red circles represent the saddle cycle $L_{-}$surrounding the stationary point $q_{-}$. The segment of the path from $A$ to $B$ is the "chaotic" life time segment (non-smooth part of the lifetime plot in figure 4.4a; refer to the text).

of the calculated MAP and it does not mean that $A=\lim _{\varepsilon \downarrow 0} A_{\varepsilon}$ since this limit does not exist and the set of the accumulation points of $\lim _{\varepsilon \downarrow 0} A_{\varepsilon}$ is the limit cycle $L_{-}$. In this sense, we can say that the invariant set $L_{-}$is the most probable exit set. Another numerical consequence of this finite arclength approximation is that the numerical value of the optimal fluctuational force is not as satisfactory at the intersection point $A$ and the final state $q_{-}$as at the saddle point $O$ (cf. figure $4.4 \mathrm{~d}$ ) because the true MAP spirals in an infinite number of loops near $A$ and $q_{+}$.

Although the calculated MAP does not automatically give the most probable invariant set, in this case the set $L_{-}$, it does provide a good approximation for the point where the most probable exit invariant intersects the MAP (here the point $A$ ). Usually, such an intersection point can be seen from the life time plot. In this case, we were able to find the limit cycle $L_{-}$using Newton's method with a good initial guess, the point $A$, and confirm that the point $A$ is on the limit cycle $L_{-}$.

The reason why the most probable invariant exit set is the one-dimensional limit cycle $L_{-}$in the three-dimensional Lorenz system is not as trivial as in planar systems where the limit cycle itself is the basin boundary. It has its origin in the qualitative behavior of the Lorenz system. In the Lorenz system, the limit cycle $L_{-}$is of saddle type and at the intersection of its two-dimensional unstable manifold $W^{u}\left(L_{-}\right)$and 


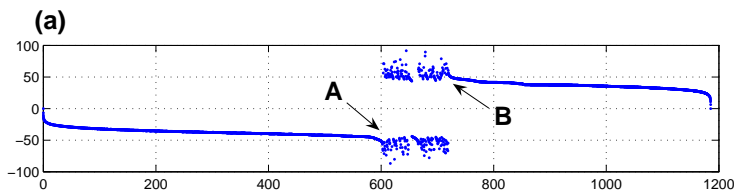

(b)

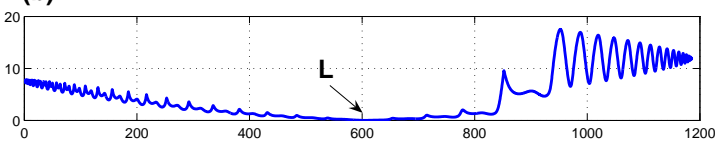

(c)

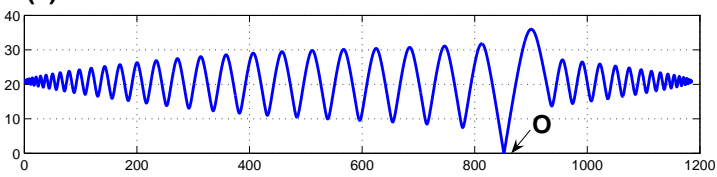

(d)

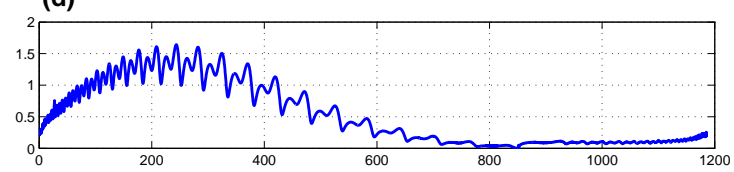

FIG. 4.4. (a) The life time plots for the MAP in figure 4.4. The horizontal axis is the arclength parameter of the MAP. The chaotic life time segment is between the labels A and B (cf. figure 4.3). (b) the Euclid distance to the limit cycle $L_{-}$from points on the MAP. The label " $L$ " indicates the position on the MAP which has the minimum distance to the limit cycle $L_{-}$. " $L$ " coincides with the point $A$ by checking their arclength parameters. (c) the Euclid distance to the origin $O$ from points on the MAP. (d) the optimal fluctuational force $\left\|\varphi^{\prime}-b(\varphi)\right\|$. The error is slightly larger near the final state $q_{+}$because the flowlines are highly spiralling and the optimization method in MAM has ill condition number near there.

stable manifold $W^{s}\left(L_{-}\right)$. The stable manifold $W^{s}\left(L_{-}\right)$forms a tube (cf. visualization in [45] ) around $q_{-}$; the unstable manifold $W^{u}\left(L_{-}\right)$inside the tube goes to the point $q_{-}$while to the chaotic set $\Lambda$ on the other side. Thus, the tube, $W^{s}\left(L_{-}\right)$, works like a separatrix. Any trajectories inside this tube are attracted toward the stationary point $q_{-}$and the life time smoothly depends on the initial condition. This part of the phase space inside $W^{s}\left(L_{-}\right)$can be viewed as a regular part of the basin of attraction of $q_{-}$. Outside of the tube lie the chaotic invariant set $\Lambda$ and the stable manifold of the origin $O$. The above discussion helps us to understand the role of $L_{-}$ in the noise-induced transition process. $L_{-}$is actually the relative attractor on the separatrix-like two-dimensional tube $W^{s}\left(L_{-}\right)$. The fluctuational noise drives most of the transition trajectories to leave the regular part, which is inside the tube, of the basin of attraction of $q_{-}$through the small neighborhood (its size is dependent on the noise intensity $\varepsilon$ ) of this limit cycle.

Outside of the limit cycle $L_{-}$lies the stable manifold of the point $O$, which separates the basins of attractions of $q_{-}$and $q_{+}$. However, this manifold $W^{s}(O)$ is not a regular two dimensional surface, but a fractal set (cf. [33]). There are an infinite number of convoluted sheets of $W^{s}(O)$ organized in a complicated way and accumulating on each other just outside the tube $W^{s}\left(L_{-}\right)$, so that $W^{s}\left(L_{-}\right)$is the $\alpha$-limit set of $W^{s}(O)$. The spacing between neighboring sheets tends to zero when approaching the limit cycle $L_{-}$. The intervals between neighboring sheets correspond 
to basins of attractions of $q_{-}$or $q_{+}$, which stagger across each other (cf. figure 4.5). Trajectories starting from different intervals between these sheets may ultimately reach either $q_{-}$or $q_{+}$. It is this structure that causes the emergence of the chaotic invariant set in the Lorenz system. [33] schematically illustrated the structure of the infinite number of sheets of $W^{s}(O)$ just outside the limit cycle $L_{-}$using numerical simulations. Since the manifold $W^{s}(O)$ asymptotically rolls onto the limit cycle $L_{-}$, there is a heteroclinic orbit starting from $L_{-}$and going to the origin $O$, which lies exactly on $W^{s}(O)$. This heteroclinic orbit is the true solution of the MAP from $L_{-}$ to $O$ and the life time for this heteroclinic orbit should be infinite since all flow lines starting from points on $W^{s}(0)$ head for the origin $O$. However, any deviation from the true solution, no matter how small, will lead to qualitative change in the life time plot since they will likely leave $W^{s}(0)$. That is the reason why the life time from $A$ to $O$ shown in figure $4.4 \mathrm{a}$ is not infinite. The appearance of the chaotic life time segment between $A$ and $B$ is due to the irregular staggering of the basins of attractions of $q_{-}$and $q_{+}$near the limit cycle $L_{-}$. The smooth part from $B$ to $O$ is due to more regular basins of attractions of $q_{-}$and $q_{+}$near the origin. Figure 4.5 illustrates the local basins of attractions around two points near $L_{-}$and near $O$, respectively. In our computations of MAP from $q_{-}$to $q_{+}$with different numerical precisions, we find that the position of the point $B$ where life time shifts from being chaotic to being regular varies largely with different numbers of grid points and transition time interval $T_{2}-T_{1}$; however the point $A$ where life time shifts from being regular to being chaotic always stay very close to or on the limit cycle $L_{-}$and the origin $O$ is always very close to the MAP. Thus the point $B$ depends on the numerical resolution in the cascade of scales in the chaotic invariant set $\Lambda$. But the limit cycle $L_{-}$and the saddle point $O$ are very stable as the transition set and transition state, respectively.

The sensitivity of the life time for the part of the MAP on the separatrix $W^{s}(O)$ reminds us to exercise caution when using life time plots to draw conclusions since it is extremely difficult to be very accurate when computing the heteroclitic orbit which lies on a fractal basin boundary. In such cases, we should combine the life time plot with other information, such as the geometric distance plots in figure $4.4 \mathrm{~b}$ and figure $4.4 \mathrm{c}$.

4.3. $\rho=\rho_{3}$ : strange attractor. When the parameter $\rho$ passes the value $\rho_{a}$, the chaotic invariant set $\Lambda$ becomes the Lorenz attractor and there is a global bifurcation that the two branches of the unstable manifolds of the origin, $\Gamma_{ \pm}$, approach toward the limit cycle $L_{\mp}$, respectively, rather than toward the nontrivial stationary points $q_{\mp}$. The Lorenz attractor is located, roughly speaking, between $\Gamma_{ \pm}$and $L_{ \pm}$ with a butterfly shape (cf. figure 2.1). But there is no qualitative change of the tube-like stable manifold of the limit cycle $L_{-}$.

The calculated MAP is shown in figure 4.6 and the related quantities are presented in figure 4.7. The MAP still crosses over $L_{-}$to escape from the basin of attraction of $q_{-}$. Now, the stable manifold of $L_{-}$separates the basin of attraction of $q_{-}$and the basin of attraction of the Lorenz attractor. Thus, trajectories starting outside of the saddle cycle $L_{-}$never settle down either on $q_{-}$or $q_{+}$, but are attracted to the Lorenz attractor. This part of the MAP corresponds to the blank interval in the life time plot of figure $4.7 \mathrm{a}$.

After crossing the saddle cycle $L_{-}$, the MAP stays in the Lorenz attractor $\Lambda$ for quite a few loops around the saddle cycle with increasing size and then heads towards the saddle point $O$. Along the unstable manifold $\Gamma_{-}$, the MAP approaches the second limit cycle $L_{+}$which is embedded in the basin boundary of $q_{+}$(cf. figure $4.7 \mathrm{~b}$ ). After 

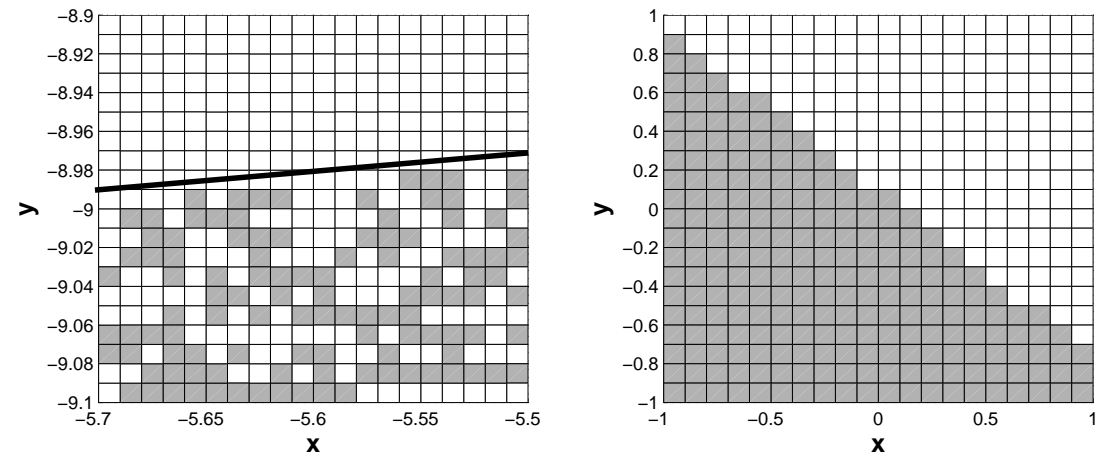

FIG. 4.5. Basins of attractions restricted to the planes $z=8.375$ (left panel) and $z=5$ (right panel). The white squares show the basin of attraction of $q_{-}$and the grey squares show that of $q_{+}$. The center of the left panel is very close to the limit cycle $L_{-}$and the dark thick line illustrates the intersection of $W^{s}\left(L_{-}\right)$with the restriction plane $z=8.375$. The white region above this dark line corresponds to one part of the basin of attraction of $q_{-}$which is inside the tube $W^{s}\left(L_{-}\right)$. The right panel corresponds to points far from $L_{-}$and near the origin $O$, where the basins of attractions of $q_{-}$and $q_{+}$become much more regular. Note that the length scale of the small square lattices in the two panels are different.

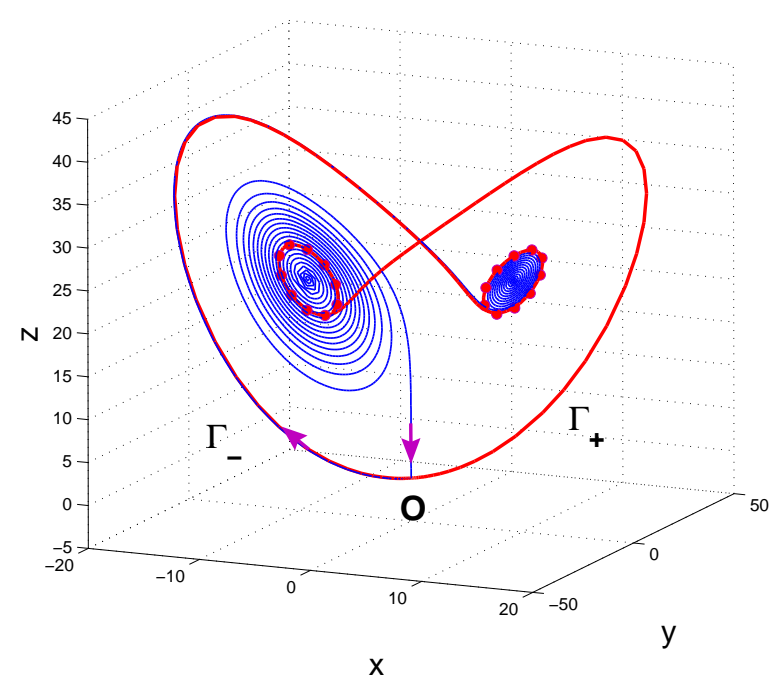

FIG. 4.6. The strange attractor case $(\rho=24.08)$ : the MAP is the blue curve. The red lines with solid dots show the unstable manifold of the origin $O$, which asymptotically approaches two saddle cycles $L_{ \pm}$(red solid circles) surrounding $q_{ \pm}$, respectively.

crossing this limit cycle $L_{+}$, the MAP goes to the final state $q_{+}$. The complete transition process involves three attractors $q_{ \pm}$and $\Lambda$ and looks in a simple way like " $q_{-} \rightarrow L_{-} \rightarrow \Lambda \rightarrow O \rightarrow L_{+} \rightarrow q_{+}$".

Since $\Lambda$ is a strange attractor, the fluctuational trajectories spend considerable amount of time wandering on the attractor before exit to $q_{+}$. The exit problem from 
(a)
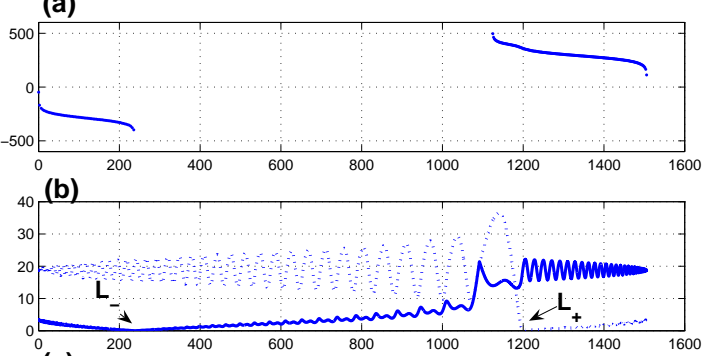

(c)
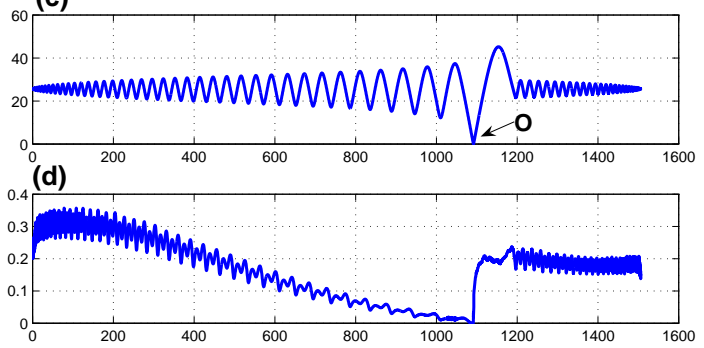

FIG. 4.7. (a) The life time plot for the MAP in figure 4.6. The horizontal axis is the arclength parameter of the MAP. The blank interval of the arclength around 225-1120 means that the trajectory goes neither to $q_{+}$nor to $q_{-}$, but to the Lorenz attractor (the life time value is thus infinite). (b) The Euclidean distance to the limit cycle $L_{-}$(solid line) and $L_{+}$(dotted line) from points on the MAP. The label " $L_{-}$" (" $L_{+}$") indicates the position on the MAP which has the minimum distance to the limit cycle $L_{-}\left(L_{+}\right)$. (c) The Euclidean distance to the origin $O$ from points on the MAP. (d) The numerical optimal fluctuational force. After the origin $O$, the numerical result deviates from the true solution (zero value) because the optimization is ill conditioned, although the geometric shape of the MAP is not distinguishable from the true solution $\Gamma_{-}$in figure 4.6.

$\Lambda$ to $q_{+}$has been investigated in [37] using Monte Carlo simulation. The authors observed enhancement of the invariant measure near the heteroclinic orbits $\Gamma_{ \pm}$with the presence of noise and the crossing of the fluctuational trajectories in transitions from the saddle cycle $L_{+}$toward $q_{+}$. The optimal transition path they obtained from the Monte Carlo simulation is consistent with the calculated MAP here. But they did not discuss the MPEP from $q_{-}$to $O$ and the importance of $L_{-}$.

Let us summarize the above three transition paradigms. They all have the saddle point $O$ as the transition state. At $\rho_{2}$ and $\rho_{3}$, the limit cycle $L_{-}$around the initial state $q_{-}$takes the additional role of the most probable exit invariant set. At $\rho_{3}$, the second limit cycle $L_{+}$shows up where the MAP enters the basin of attraction of $q_{+}$.

\section{Discussion}

The example of the Lorenz system demonstrates the possibility that limit cycles of saddle type, the next simplest invariant sets beyond fixed points, determines the most probable exit locations on the basin boundary in the fluctuational transition. Moreover, although both the transient chaos and the Lorenz attractor have complicated dynamical structures, the transition process can be understood in a much simpler way as long as we identify the saddle cycle $L_{-}$and the saddle point $O$ as transition sets. The saddle cycle $L_{-}$delimitates the basin of attraction of $q_{-}$with the chaotic set and the saddle point $O$ separates the chaotic set from the basin of attraction of $q_{+}$. These 
two simple structures function like two gateways on the road of transition processes from $q_{-}$to $q_{+}$. The observed fluctuational trajectories in experiments typically goes through these gateways successively along the MAP. These findings might be relevant for other non-gradient systems.

Acknowledgement. We are grateful to Phil Holmes and one of the anonymous referees for very helpful discussions.

\section{REFERENCES}

[1] H.A. Karmers, Brownian motion in a field of force and the diffusion model of chemical reactions, Physica, 7, 284-304, 1940

[2] W. Ren, Numerical methods for the study of energy landscape and rare events, PhD thesis, New York University, 2002

[3] W. E, W. Ren and E. Vanden-Eijnden, String method for the study of rare events, Phys. Rev. B, 66, 052301, 2002 .

[4] W. E, W. Ren and E. Vanden-Eijnden, Simplified and improved string method for computing the minimum energy paths in barrier-crossing events, J. Chem. Phys., 126, 164103, 2007.

[5] M.H. Devoret, D. Esteve, J.M. Martinis, A. Cleland and J. Clarke, Resonant activation of a Brownian particle out of a potential well: microwave-enhanced escape from the zero-voltage state of a Josephson junction, Phys. Rev. B, 36(1), 58-73, 1987.

[6] J. Hales, A. Zhukov, R. Roy and M.I. Dykman, Dynamics of activated escape and its observation in a semiconductor laser, Phys. Rev. Lett., 85(1), 78-81, 2000.

[7] A. Sali, E. Shakhnovich and M. Karplus, How does a protein fold, Nature, 369, 248-251, 1994.

[8] D.G. Luchinsky and P.V.E. McClintock, Irreversibility of classical fluctuations studied in analogue electrical circuits, Nature, 389, 463-466, 1997.

[9] R.S. Maier and D.L. Stein, Transition-rate theory for nongradient drift fields, Phys. Rev. E, 69(26), 3691, 1992

[10] R.S. Maier and D.L. Stein, Effect of focusing and caustics on exit phenomena in systems lacking detailed balance, Phys. Rev. Lett., 71(12), 1783, 1993.

[11] N. Berglund and B. Gentz, On the noise-induced passage through an unstable periodic orbit I: two-level model, J. Stat. Phys, 114(5), 1577-1618, 2004.

[12] L. Onsager and S. Machlup, Fluctuations and irreversible processes, Phys. Rev., 91, 1505-1512, 1953.

[13] R.S. Maier and D.L. Stein, Escape problem for irreversible systems, Phys. Rev. E, 48(2), 931938, 1993

[14] R.L. Kautz, Activation energy for thermally induced escape from a basin of attraction, Phys. Rev. A, 125, 315-319, 1987.

[15] R.L. Kautz, Thermally induced escape: the principle of minimum available noise energy, Phys. Rev. A, 38(4), 2066-2080, 1988.

[16] S. Kraut and C. Grebogi, Escaping from nonhyperbolic chaotic attractors, Phys. Rev. Lett., 92(23), 234101, 2004.

[17] D.G. Luchinsky and I.A. Khonanov, Fluctuation-induced escape from the basin of attraction of a quasiattractor, JETP Letters, 69(11), 825-830, 1999.

[18] I.A. Khonanov, D.G. Luchinsky, R. Mannella and P.E. McClintock, Fluctuation and the energyoptimal control of chaos, Phys. Rev. Lett., 85(10), 2100, 2000.

[19] D.G. Luchinsky, S. Beri, R. Mannella and P.V. McClintock, Optimal fluctuations and the control of chaos, Internat. J. Bifur. Chaos Appl. Sci. Engrg., 12(3), 583-604, 2002.

[20] A.N. Silchenko, D.G. Luchinsky and P.V.E. McClintock, Noise-induced escape through a fractal basin boundary, Physica A, 327, 371-377, 2003.

[21] A.N. Silchenko, S. Beri, D.G. Luchinsky and P.V.E. McClintock, Fluctuational transitions through a fractal basin boundary, Phys. Rev. Lett., 91(17), 174104, 2003.

[22] A.N. Silchenko, S. Beri, D.G. Luchinsky and P.V.E. McClintock, Fluctuational transitions across different kinds of fractal basin boundaries, Phys. Rev. E, 71(4), 046203, 2005.

[23] W. E, W. Ren and E. Vanden-Eijnden, Minimum action method for the study of rare events, Commun. Pure Appl. Math., 57, 637-656, 2004.

[24] E. Vanden-Eijnden and M. Heymann, The geometric minimum action method for computing minimum energy paths, J. Chem. Phys., 128, 061103, 2008.

[25] M.I. Freidlin and A.D. Wentzell, Random Perturbations of Dynamical Systems, Grundlehren der mathematischen Wissenschaften. Springer-Verlag, New York, 1998. 
[26] J.L. Kaplan and J.A. Yorke, Preturbulence, a regime observed in a fluid flow model of Lorenz, Comm. Math. Phys., 67, 93-108, 1979

[27] C. Sparrow, The Lorenz Equations, Bifurcations, Chaos, and Strange Attractors, Appl. Math. Sci., Springer-Verlag, New York, 1982.

[28] J. Guckenheimer and P. Holmes, Nonlinear Oscillations, Dynamical Systems, and Bifurcation of Vecor Fields, Appl. Math. Sci., Springer, New York, 1983.

[29] J. Guckenheimer, The Hopf bifurcation and its applications, J.E. Marsden and M. McCracken (Eds.), Springer-Verlag, New York, Heidelberg, Berlin, 368-381, 1976.

[30] J. Guckenheime and R.F. Williams, Structural stability of Lorenz attractors, Publ. Math. IHES., 50, 59-72, 1979.

[31] R.F. Williams, The structural of Lorenz attractors, Publ. Math. IHES., 50, 101-152, 1979.

[32] E.A. Jackson, The Lorenz system: I. the global structure of its stable manifolds, Physica Scripta, 32, 469-475, 1985.

[33] E.A. Jackson, The Lorenz system: II. the global structure of its stable manifolds, Physica Scripta, 32, 476-481, 1985.

[34] B. Krauskopf and H.M. Osinga, The Lorenz manifold as a collection of geodesic level sets, Nonlinearity, 17, C1-C6, 2004.

[35] E.J. Doedel, B. Krauskopf and H.M. Osinga, Global bifurcations of the Lorenz manifold, Nonlinearity, 19, 2947-2972, 2006.

[36] M.I. Dykman, P.V.E. McClintock, V.N. Smelyanski, N.D. Stein and N.G. Stocks, Optimal paths and the prehistory problem for large fluctuations in noise-driven system, Phys. Rev. Lett., 68(18), 2718, 1992.

[37] V.S. Anishchenko, D.G. Lunchiinsky, P.V.E. McClintock, I.A. Khovanov and N.A. Khovanova, Fluctuational escape from a quasi-hyperbolic attractor in the Lorenz system, JETP Letters, 94(4), 821-833, 2002.

[38] D.G. Luchinsky, A.N. Silchenko, S. Beri, R. Mannella and P.V.E. McClintock, Solution of the boundary value problem for optimal escape in continuous stochastic systems and maps, Phys. Rev. E, 72, 036131, 2005.

[39] X. Zhou, W. Ren and W.E., Adaptive minimum action method for the study of rare events, J. Chem. Phys., 128(10), 104111, 2008.

[40] M. Heymann and E. Vanden-Eijnden, The geometric minimum action method: a least action principle on the space of curves, Commun. Pure Appl. Math., 61, 1052-1117, 2007.

[41] R.S. Maier and D.L. Stein, Limiting exit location distributions in the stochastic exit problem, SIAM J. Appl. Dyn. Syst., 57(3), 752-790, 1997.

[42] T.S. Parker and L.O. Chua, Practical Numerical Algorithms for Chaotic Systems, SpringerVerlag, 1989.

[43] R.S. Maier and D.L. Stein, Oscillatory behavior of the rate of escape through an unstable limit cycle, Phys. Rev. Lett., 77, 4860-4863, 1996.

[44] A. Bandrivskyy, S. Beri, D.G. Luchinsky and P.V.E. McClintock, Singularities in far-fromequilibrium distributions at finite noise intensities, S.M. Bezrukov (Eds.), Unsolved Problems of Noise and Fluctuations in Physics, Biology and High Technology, proceedings of the conference in Bethesda, American Institute of Physics, September 2002, Melville, NY, $451,2003$.

[45] M.E. Jonhson, M.S. Jolly and I.G. Kevrekidis, Two-dimensional invariant manifolds and global bifurcations: some approximation and visualization studies, Numer. Algorithms, 14, 125$140,1997$. 